\title{
Revisiting the changes in the Banff Classification for antibody-mediated rejection after kidney transplantation
}

Jasper Callemeyn ${ }^{1,2}$, Heleen Ameye ${ }^{1}$, Evelyne Lerut ${ }^{3 *}$, Aleksandar Senev ${ }^{2,4}$, Maarten Coemans ${ }^{2}$, Elisabet

Van Loon ${ }^{1,2}$, Ben Sprangers ${ }^{1,2}$, Vicky Van Sandt ${ }^{3}$, Maud Rabeyrin ${ }^{5}$, Valerie Dubois ${ }^{6}$, Olivier Thaunat ${ }^{7,8}$, Dirk Kuypers ${ }^{1,2}$, Marie-Paule Emonds ${ }^{2,4}$, Maarten Naesens ${ }^{1,2}$

Jasper Callemeyn and Heleen Ameye contributed equally to this manuscript.

${ }^{1}$ Department of Nephrology and Renal Transplantation, University Hospitals Leuven, Leuven, Belgium

${ }^{2}$ Department of Microbiology, Immunology and Transplantation, KU Leuven, Leuven, Belgium

${ }^{3}$ Histocompatibility and Immunogenetics Laboratory, Belgian Red Cross-Flanders, Mechelen, Belgium

${ }^{4}$ Department of Pathology, University Hospitals Leuven, Leuven, Belgium

${ }^{5}$ Hospices Civils de Lyon, Department of Pathology, Bron, France

${ }^{6}$ French National Blood Service (EFS), HLA Laboratory, Décines-Charpieu, France

${ }^{7}$ French National Institute of Health and Medical Research (Inserm) Unit 1111, Lyon, France

${ }^{8}$ Hospices Civils de Lyon, Edouard Herriot Hospital, Department of Transplantation, Nephrology and Clinical Immunology, Lyon, France

\section{Correspondence}

\section{Maarten Naesens}

Email: maarten.naesens@kuleuven.be

This article has been accepted for publication and undergone full peer review but has not been through the copyediting, typesetting, pagination and proofreading process, which may lead to differences between this version and the Version of Record. Please cite this article as doi: $\underline{10.1111 / \text { AJT.16474 }}$

This article is protected by copyright. All rights reserved 


\section{Abbreviations}

- ABMR: antibody-mediated rejection

- Banff'01: Banff classification in 2001

- Banff'13: Banff classification in 2013

- Banff'17: Banff classification in 2017

- Banff'19: Banff classification in 2019

- CS: corticosteroids

- DSA: donor-specific antibodies

- ENDATs: endothelial cell-associated transcripts

- g: glomerulitis

- HLA: Human Leukocyte Antigen

- i: inflammation

- IF: immunofluorescence

- IHC: immunohistochemistry

- MFI: median fluorescence intensity

- MPA: mycophenolic acid

- MVI: microvascular inflammation

- No ABMR: absence of antibody-mediated rejection

- ptc: peritubular capillaritis

- PTCs: peritubular capillaries

- SAB: single antigen beads

- sABMR: suspicious for antibody-mediated rejection

- SD: standard deviation

- $\quad$ t: tubulitis

- TAC: tacrolimus

- TCMR: T-cell mediated rejection

- $\quad$ v: arteritis 


\section{Abstract}

The Banff classification for antibody-mediated rejection (ABMR) has undergone important changes, mainly by inclusion of C4d-negative ABMR in Banff'13 and elimination of suspicious ABMR (sABMR) with the use of C4d as surrogate for HLA-DSA in Banff'17. We aimed to evaluate the numerical and prognostic repercussions of these changes in a single-centre cohort study of 949 single kidney transplantations, comprising 3662 biopsies that were classified according to the different versions of the Banff classification. Overall, the number of ABMR and sABMR cases increased from Banff'01 to Banff'13. In Banff'17, 248/292 sABMR biopsies were reclassified to No ABMR, and 44/292 to ABMR. However, reclassified sABMR biopsies had worse and better outcome than No ABMR and ABMR, which was mainly driven by the presence of microvascular inflammation and absence of HLA-DSA, respectively. Consequently, the discriminative performance for allograft failure was lowest in Banff'17, and highest in Banff' 13. Our data suggest that the clinical and histological heterogeneity of ABMR is inadequately represented in a binary classification system. This study provides a framework to evaluate updates of the Banff classification and assess the impact of proposed changes on the number of cases and risk stratification. Two alternative classifications introducing an intermediate category are explored. 


\section{Introduction}

Currently available immunosuppressive agents are able to prevent and treat T-cell mediated rejection (TCMR) but are less potent for antibody-mediated rejection (ABMR), which is considered a major cause of graft failure. ${ }^{1-3}$ In the search for potential treatments and personalized prognostication, a clear definition of $\mathrm{ABMR}$ is an indispensable prerequisite.

The Banff consortium has provided an internationally adopted classification for acute/active ABMR. First established in 2001, three diagnostic criteria were outlined. ${ }^{4,5}$ First, morphologic evidence of acute tissue injury was defined as 'neutrophils and/or monocytes/macrophages in peritubular or glomerular capillaries ( $\mathrm{ptc}>0$ or $\mathrm{g}>0$ ), arterial fibrinoid necrosis $(\mathrm{v}=3$ ), thrombotic microangiopathy (TMA), or acute tubular injury. Second, evidence of antibody-endothelium interaction required positive $\mathrm{C} 4 \mathrm{~d}$ staining in peritubular capillaries, defined since 2007 as grade 2 or higher ( $\geq 10 \%$ ) on immunohistochemistry (IHC) or grade 3 on immunofluorescence (IF) ( $\geq 50 \%$ ). ${ }^{6,7}$ Finally, serologic evidence was defined as circulating antibodies to donor HLA or other donor-specific antibodies (DSA). In case of morphologic and immunohistologic evidence without serologic evidence, or in case of morphologic and serologic evidence without immunohistologic evidence, biopsies were classified as 'suspicious for ABMR' (sABMR). ${ }^{5}$

In Banff' 13 the threshold for arteritis was lowered to $\mathrm{v}>0$, and exclusion of glomerulonephritis was required in case of glomerulitis. ${ }^{6,8-10}$ The immunohistologic criterion was broadened to linear $\mathrm{C} 4 \mathrm{~d}$ staining of peritubular capillaries, with a IHC cutoff of $>0 \%$ and IF $>10 \%,{ }^{11}$ or presence of at least moderate microvascular inflammation (MVI, defined as $\mathrm{g}+\mathrm{ptc} \geq 2$ ), ${ }^{12-14}$ thus acknowledging the presence of C4dnegative ABMR. Isolated peritubular capillaritis without glomerulitis was not considered as MVI in the presence of TCMR, borderline changes, or infection. Increased expression of transcripts associated with endothelial injury (ENDATs) was incorporated into the second criterion as well. ${ }^{15}$

In Banff'17, the classification for acute/active ABMR was importantly revised a second time. First, the exclusion of isolated ptc in the presence of TCMR or borderline changes or infection was introduced in the morphologic criterion. ${ }^{16}$ Second, increased gene expression was broadened from ENDATs to all validated ABMR-associated genes. ${ }^{16,17}$ Finally, C4d staining and increased gene expression were accepted as substitutes for DSA in the serological criterion for DSA negative cases. ${ }^{16-18}$ Importantly, by the incorporation of a large part of the second criterion into the third criterion, the suspicious category was eliminated in the Banff' 17 revision. The criteria for ABMR were unchanged in Banff' $19 .{ }^{19}$

As the Banff classification changes are implemented in routine clinical practice worldwide, all of these changes have affected patients' clinical trajectories and medical decisions. However, it is unclear to what extent these changes have numerically influenced the categorization of kidney transplant biopsies, and whether these changes have contributed to better risk stratification. Therefore, we assessed and validated 
the impact of previous Banff revisions on the prevalence and prognosis of ABMR diagnostic categories, and provide a framework in which potential future Banff revisions can be evaluated.

This article is protected by copyright. All rights reserved 


\section{Methods}

\subsection{Study population and data collection}

All consecutive kidney transplantations in adult recipients at the University Hospitals Leuven between March 2004 and February 2013 were considered eligible. Combined transplantations, kidney transplantation after another solid organ transplant, and transplantations without biopsy follow-up were excluded. Allograft biopsies were performed at protocol biopsy time points $(3,12,24$, and sometimes at 36 , 48 and 60 months after transplantation), in addition to clinically indicated biopsies. All clinicopathological data were prospectively collected during routine clinical follow-up. This study was approved by the Ethics Committee of the University Hospitals Leuven (S53364 and S61788).

The independent validation cohort consisted of allograft biopsies, performed either for cause or as part of the routine follow-up at 3 months and 12 months post transplantation, from all consecutive adult kidney transplantations between January 2007 and December 2015 at Hospices Civils de Lyon, France.

\subsection{Clinicopathological assessment}

All post-transplantation biopsies were reviewed retrospectively by a single pathologist (EL). The severity of individual histological lesions was scored semiquantitatively according to the Banff criteria. ${ }^{20}$ Biopsies that were considered as inadequate or lacked scoring of the relevant individual lesions were excluded from the analyses. Based on the individual lesions, each biopsy was classified according to the international Banff classification for ABMR in the Banff'014, Banff' $13^{9}$ and Banff' $17^{16}$ editions, respectively. For Banff'01, documentation of immunoglobulin or complement in arterial fibrinoid necrosis was not included, since this was not systematically assessed in all biopsies. Acute tubular injury was not taken into account given the absence of sufficient clinical information to exclude other causes. In case of microvascular inflammation, de novo or recurrent glomerulonephritis was not used as exclusion factor in our study. For C4d deposition, an immunohistochemical staining (monoclonal antibody, dilution 1:500; Quidel Corporation, Santa Clara, CA) on frozen tissue was performed. C4d deposition in peritubular capillaries (PTCs) was scored as following: C4d0-no staining ( $0 \%)$, C4d1-minimal staining $(>0 \%<25 \%$ of PTCs), C4d2-focal staining (25-75\% of PTCs), and C4d3-diffuse staining ( $>75 \%$ of PTCs). A cut-off value of C4d $\geq 2$ was considered positive. ${ }^{21}$ For the Lyon validation cohort, all post-transplant biopsies were scored by the pathologist at the time of the biopsy, including evaluation of the individual Banff lesions. In addition to acute tubular injury and glomerulonephritis, information on thrombotic microangiopathy was not available.

\subsection{Detection of circulating anti-HLA donor-specific antibodies}

The retrospective HLA-DSA reassessment on prospectively biobanked serum samples has been outlined previously. ${ }^{21}$ Briefly, pre- and posttransplant anti-HLA antibodies were systematically tested at the time of 
each biopsy irrespective of the histological picture, using a sensitive Luminex-based assay in 1 histocompatibility laboratory (HILA-Red Cross- Flanders). Antibodies against HLA-A, -B, -C, -DRB1, -DRB345, -DQ, and -DP loci in the recipient sera were determined for donor specificity at the resolution level of single antigen beads (SAB). High-resolution typing of donors and recipients was performed to determine true donor specificity. ${ }^{22}$ A positive result for circulating HLA-DSA was defined as a median fluorescence intensity (MFI) of the donor-specific bead above 500. At the time of an allograft biopsy, HLA-DSA positivity was determined by presence of pretransplant HLA-DSA and/or documentation of de novo HLA-DSA during follow-up after transplantation. For 113/3622 biopsies (3.1\%), no sera were available, and in these cases results from the previous measurements were extrapolated.

\subsection{Statistical analysis}

Kidney allograft survival was plotted using Kaplan-Meier survival curves, and survival between groups was compared using log-rank testing, with Bonferroni correction for pairwise comparisons. Graft survival was censored at the time of death in case of death with a functioning graft. Univariable and multivariable Cox models were applied to quantify the hazard ratios for allograft failure. C-statistics were performed to assess the prognostic ability of different classifications for allograft outcome, and $95 \%$ confidence intervals were calculated using 1000-fold bootstrapping. Two-sided P-values less than 0.05 were considered statistically significant. We used SAS Software for statistical analysis (version 9.4, SAS Institute Inc., Cary, NC, United States), and GraphPad Prism for graphical presentation (version 8.0.1, GraphPad Software,

San

Diego,

CA,

United

States). 


\section{Results}

\subsection{Study population characteristics}

The study cohort consisted of 949 transplantations, with a median follow-up of 7.5 years after transplantation (IQR 4.9-10.0). Clinical characteristics are represented in Table 1. In total, 3622 biopsies were performed, comprising 2775 protocol and 847 indication biopsies.

\subsection{Evolution of SABMR and ABMR diagnosis between Banff editions}

Of the 3662 included biopsies, 491 biopsies were classified as ABMR or sABMR by at least one edition of the Banff classification (Figure 1, Table 2).

According to Banff'01, 74/491 (15.1\%) were classified as ABMR, 238/491 (48.5\%) as sABMR and 179/491 (36.5\%) as No ABMR. Comparing Banff'13 with Banff'01, four Banff'01 No ABMR biopsies were reclassified as ABMR by Banff' 13 , because of the new criterion for morphologic evidence ( $\mathrm{v}>0$ instead of $\mathrm{v}=3$ ). Of the Banff'01 No ABMR biopsies, 175 were reclassified as sABMR by Banff'13: 10/175 (5.7\%) because of morphologic and serologic evidence ( $v>0$ and HLA-DSA positivity), and $165 / 175$ (94.3\%) because of morphologic and immunohistologic evidence ( 4 because of $v>0$ and $C 4 d$ positivity; 161 because of at least moderate MVI).

Of the Banff'01 sABMR biopsies, 121/238 (50.8\%) C4d negative sABMR biopsies with at least moderate MVI were reclassified as ABMR. Thus, mainly due to the use of at least moderate MVI as second criterion, Banff' 13 contained the largest number of biopsies diagnosed with sABMR or ABMR ( $n=292$ and n=199, respectively).

As the suspicious category was eliminated in Banff'17, 248/292 (84.9\%) Banff' 13 sABMR biopsies were reclassified as No ABMR in Banff' 17, 79/248 (31.9\%) due to absence of the second criterion (e.g. C4d negative, HLA-DSA positive arteritis without MVI), 161/248 (64.9\%) due to absence of the third criterion (i.e. C4d negative, HLA-DSA negative with MVI) and 8/248 (3.2\%) were excluded due to borderline changes or TCMR concomitant with isolated ptc. The other 44/292 (15.1\%) Banff' 13 sABMR biopsies were reclassified as ABMR in Banff' 17, because of positive C4d staining in the absence of HLA-DSA. Of the 199 Banff'13 ABMR biopsies, 6/199 (3.0\%) were reclassified to No ABMR in Banff'17 because of cellular rejection in combination with isolated ptc, whereas 193/199 (97.0\%) remained classified as ABMR in Banff' 17.

\subsection{Comparison of predictive performance for allograft survival between Banff editions}

In order to assess whether the evolving definitions of ABMR impacted the prognostication of allograft outcome, we performed a landmark analysis of death-censored allograft survival after the first posttransplant year ( $\mathrm{N}=893$ transplantations with a functioning transplant). Transplantations were stratified 
according to the most severe rejection category (in decreasing order of severity; ABMR, sABMR and No ABMR) that was diagnosed within the first year. In the sABMR group, 75/114 were derived from indication biopsies, and 47/70 ABMR cases were derived from indication biopsies. Comparing Banff'01 and Banff'13, the hazard ratio for allograft failure after ABMR increased from 3.87 (95\% CI 2.00-7.51) to 4.39 (95\% CI 2.67-7.22). In contrast, the risk after ABMR defined according to Banff' 17 was lower (HR 3.54, 95\% CI 2.27-5.54) (Fig 2A). A landmark analysis combining the sABMR and ABMR category in Banff'01 and Banff'13 is provided in Fig S1. We also calculated the Harrell's C-index for each Banff version at the time of the first indication biopsy, and protocol biopsies performed at three months, one year and two year after transplantation (Fig 2B). Across all protocol biopsy moments, Banff'17 had the lowest discriminating performance for death-censored allograft failure, whereas Banff' 13 performed best.

To address the cause of these prognostic differences between Banff editions, we categorized biopsies according to their diagnosis across classifications (e.g. Banff'01 sABMR $\rightarrow$ Banff'13 ABMR vs. Banff'01 $\mathrm{ABMR} \rightarrow$ Banff'13 ABMR) in a landmark analysis after the first posttransplant year (Fig 3-4). Comparing Banff'01 and Banff'13, cases reclassified from No ABMR to sABMR were associated with worse survival than biopsies that were considered No ABMR in both editions (Bonferroni-adjusted $P<0.001$ ), whereas sABMR reclassified to ABMR had a similar outcome as biopsies considered as ABMR in both editions (adjusted $P=0.68$ ), illustrating the improved risk stratification of Banff' 13 in comparison to Banff'01. In contrast, Banff'13 sABMR cases reclassified to No ABMR in Banff' 17 conferred worse graft survival than biopsies considered as No ABMR in both editions (adjusted $P<0.001$ ), whereas those sABMR cases reclassified to ABMR in Banff' 17 had a significantly better outcome than cases defined as ABMR in both editions (adjusted $P<0.001$ ). This illustrates that the reclassification of cases in Banff' 17 did not contribute to improved risk stratification in comparison to Banff' 13 . In a sensitivity analysis correcting for biopsy timing, context and transplantation period, these results remained consistent, although power was lacking in some groups at later biopsy moments (Figure S2-3).

As shown in Table S1, some ABMR or sABMR cases had concomitant transplant glomerulopathy (cg) or cellular rejection (borderline changes or TCMR), and varying degrees of IFTA at the time of diagnosis. In order to assess the impact of these concomitant lesions and phenotypes at different time points and clinical contexts, we performed a multivariable analysis incorporating TCMR, cg score and IFTA grade together with the evolution between Banff' 13 and Banff' 17 versions, for protocol biopsies performed at 3 months, 1 year and 2 years after transplantation, and at the first indication biopsy (Table 3). Our main finding that the allograft prognosis of Banff'13 sABMR $\rightarrow$ Banff'17 No ABMR is significantly worse than No ABMR'13 $\rightarrow$ No ABMR'17 remained consistent across all protocol biopsy moments. In the indication biopsies, only ABMR that was consistently diagnosed across 2013 and 2017 classifications had significantly worse outcome.

This article is protected by copyright. All rights reserved 
An overview of the administered treatment of the 491 biopsies considered as sABMR or ABMR in Banff' 13 is provided in Table S1. Specific treatment was administered in 54/491 cases (11.0\%). The treatment options consisted of ATG, plasmapheresis, bortezomib, rituximab and IVIG. Most patients received monotherapy ( $n=26$ ATG, $n=10$ plasmapheresis, $n=3$ bortezomib), whereas 15 others received varying treatment combinations. Comparing Banff' 13 sABMR and Banff' 13 ABMR, there was no difference in the number of patients receiving specific antibody-targeted therapy ( $9.2 \%$ vs. $13.5 \%)$ or highdose steroids (32.5\% vs. $34.7 \%$ ). Importantly, ABMR specific therapy was only administered in the context of an indication biopsy. Within Banff' 13 sABMR, there were no differences in administration of specific therapy among cases that were classified to either No ABMR or ABMR in Banff' 17 (8.8\% vs. 11.4\%), although the latter group received high-dose steroids more often $(29.4 \%$ vs. $50.0 \%)$. sABMR and ABMR in transplantations performed between 2004 and 2008 were treated less frequently with specific ABMR treatment compared to kidneys transplanted after 2008 (5.9\% vs. $13.8 \%$, and $7.2 \%$ vs. $21.6 \%$, Table S2). In a multivariable analysis of the 126 first indication biopsies with Banff' 13 sABMR or Banff' 13 ABMR, incorporating specific treatment, high-dose steroids, transplantation period and post-transplant time, neither treatment modalities nor transplantation period accounted for survival differences between the histological categories, although numbers were too small to draw definitive conclusions (Table S3).

Next, we sought to determine which of the diagnostic Banff criteria were contributing to the perceived survival differences between groups. We performed a multivariable analysis incorporating MVI score, HLA-DSA status, and C4d score (Table S4). Across different time points, with the exception of three months post-transplant, HLA-DSA independently associated with worse allograft outcome, whereas MVI severity had a negative prognostic effect at three months and one year post-transplant. Importantly, C4d score was not informative to predict allograft outcome. Taken together, differences in outcome were mainly driven by the absence of HLA-DSA in Banff' 13 sABMR $\rightarrow$ Banff' 17 ABMR, and the presence of MVI in Banff' 13 sABMR $\rightarrow$ Banff' 17 No ABMR.

Given the importance of HLA-DSA and MVI, we explored whether the addition of an intermediate category consisting of either of these parameters, could improve the prognostic performance of the Banff classification. In a first alternative approach, we reserved the term "ABMR" only for those DSA positive biopsies that were considered as ABMR'17, and introduced a new category termed "DSA negative MVI". An overview of the numerical impact of these changes is given in Figure S4 and Table S5. In a landmark analysis after the first year, DSA negative MVI associated with an increased risk of allograft failure (HR $1.92,95 \%$ 1.08-3.42, Figure 5A,C). In comparison with Banff' 17 , the concordance index improved at 3 months and 1 year post-transplant (Fig 5B). In a second approach, we added a category termed "DSA positive suspicious" for HLA-DSA positive biopsies with lesions meeting the first criterion (i.e. $\mathrm{g}+\mathrm{ptc}=1$, $\mathrm{v}>0$ or TMA) but not the second Banff' 17 criterion (Figure S5, Table S5). Although the numbers were too 
small to reach statistical significance in the 1 year landmark analysis, the concordance index was further improved by this addition, especially at 2 years after transplantation (Fig 5A-B,D).

\subsection{Independent validation of the findings}

Finally, we evaluated whether our findings could be replicated in an independent cohort of 1609 allograft biopsies from 725 kidney transplantations performed at Hospices Civils de Lyon, with a median follow-up of 5.4 years (IQR 4.0-7.6). Clinical characteristics of the cohort are available in Table S6. In contrast with our study cohort, none of the recipients had pre-transplant HLA-DSA, although a larger proportion (90/725, 12.4\%) developed de novo HLA-DSA after transplantation. As a result, a higher fraction of HLA-DSA negativity in MVI biopsies was reported, compared to our cohort (66\% vs. 51.5\%), and ABMR or sABMR according to Banff' 13 tended to occur later in the post-transplant period (median 356 days, IQR 114-527 vs. 92 days, 8-381). Since C4d deposition was more frequently observed in HLA-DSA positive MVI than HLA-DSA negative MVI, Banff' 13 caused a minor increase of 12 ABMR cases, whereas 136 sABMR cases were added (Figure S6). In line with our results, biopsies reclassified from No ABMR to sABMR in Banff'13 had worse outcome than biopsies considered as No ABMR in both editions (Figure S7). In the sABMR group, 49/97 were derived from indication biopsies, and 7/10 ABMR cases were derived from indication biopsies. Conversely, in Banff' 17, sABMR reclassified to No ABMR conferred worse allograft survival, which did not differ from sABMR reclassified to ABMR (Figure S8), again confirming our findings. 


\section{Discussion}

Since its introduction in 2001, the Banff definition of ABMR has undergone significant revisions, mainly by inclusion of C4d-negative ABMR in Banff'13 and removal of the suspicious category with the use of C4d deposition as a surrogate for HLA-DSA in Banff'17. This study confirms that these modifications have drastically impacted the number of ABMR diagnoses, which sequentially increased after each iteration, although the prognostic performance for allograft outcome declined in Banff' 17.

In Banff' 13, ABMR and sABMR numbers increased mainly due to the inclusion of MVI as surrogate for C4d deposition in the second criterion, and, to a lesser extent, the lowered cut-off value for arteritis. Supporting these changes, allograft outcome was worse in patients reclassified from No ABMR in Banff'01 to sABMR in Banff'13, in comparison to patients where the diagnosis remained No ABMR. Moreover, graft outcome was similar in biopsies classified as ABMR in Banff'13, regardless of the classification in Banff'01.

As the sABMR category was eliminated in Banff' $17,15 \%$ of sABMR cases were reclassified to ABMR. However, these reclassified cases (i.e. HLA-DSA negative, C4d positive ABMR) had a significantly better outcome than HLA-DSA positive ABMR, confirming our earlier findings that HLA-DSA are a negative prognostic factor. ${ }^{23}$ In protocol biopsies, sABMR cases in Banff' 13 reclassified to No ABMR in Banff' 17 had worse allograft survival, compared to those already classified as No ABMR in Banff' 13 . In indication biopsies, only ABMR that was consistently diagnosed across Banff' 13 and Banff' 17 classifications (i.e. HLA-DSA positive cases) had significantly worse outcome. In a multivariable model, we demonstrated that MVI severity and HLA-DSA independently associate with allograft outcome and largely explain the detected prognostic differences. Importantly, although $\mathrm{C} 4 \mathrm{~d}$ is used a surrogate criterion for HLA-DSA in Banff' 17, C4d severity did not increase the risk for allograft failure.

In an independent validation cohort, we confirmed that a numerous fraction of sABMR biopsies was reclassified to No ABMR in Banff'17, yet associated with worse allograft outcome. Importantly, this population had a lower immunological risk, as none of the recipients had pretransplant HLA-DSA, which was reflected by the later onset of ABMR or SABMR. Our conclusions thus appear to be consistent across transplant populations with different immunological risks and timing of rejection.

Given that the large majority of patients with sABMR or ABMR did not receive specific therapy, our study allows for a better understanding of the natural disease course of these rejection phenotypes. Since sABMR associates with an intermediate prognosis compared to ABMR and No ABMR, eliminating the suspicious category may have led to under- or overtreatment of sABMR patients that were recategorized in Banff' 17 to No ABMR or ABMR, respectively. Taken together, these data suggest that the current Banff'17 binary definition of ABMR oversimplifies the apparent heterogeneous clinical and histological picture of this 
disease. Instead, we argue that intermediate cases could be represented by the reintroduction of a dedicated category, such as "DSA negative MVI", which might also include C4d+DSA-MVI cases. In an exploratory analysis, the addition of a "DSA negative MVI" category increased the predictive performance, as well as the inclusion of a separate "DSA positive suspicious" category. Alternatively, the incorporation of continuous variables such as MVI severity could shift the classification towards a probabilistic approach and provide more granular insights. Multicenter analyses will be necessary to define the optimal ensemble of diagnostic criteria for the spectrum of MVI.

The Banff criteria are applied every day in transplant clinics worldwide for treatment decisions, and are a cornerstone in the development of clinical trials and approval of therapeutic interventions. ${ }^{24}$ In addition to the potential clinical harm, rapidly changing definitions impede direct comparison of studies performed within less than a decade's time. Moreover, the addition of gene transcripts as a surrogate marker for HLADSA in Banff 2017 has not led to a change in clinical practice, given the absence of a consensus gene panel on a standardized molecular diagnostics platform with validated cut-off values. Therefore, as clinics and regulatory agencies watch closely, the Banff community should caution against implementing frequent modifications of the classification system, if only affecting a very small number of cases, lacking interpretability or without improving the risk stratification. Our study illustrates that the impact of future updates on prevalence and allograft prognostication could be assessed on retrospective databases.

This study has several limitations. Although all biopsies were scored by the same pathologist in this singlecenter longitudinal study, validation from other centers with a different case mix and higher risk patients will be necessary to support our findings. Given that some findings are specific for either protocol or indication biopsies, part of our results could be less applicable in transplant centers that adhere to different biopsy practices. In addition, we were unable to study all parameters used in the different Banff updates, either due to lack of systematic assessment, in case of molecular markers or immunoglobulin staining in fibrinoid necrosis, or insufficient availability of clinical information, in case of acute tubular injury or glomerulonephritis. C4d staining is performed at our center by immunohistochemistry on frozen sections, and is scored differently than by Banff standards, determining C4d positivity at $>25 \%$ of peritubular capillaries. With the use of a lower threshold, more HLA-DSA negative biopsies would be included in the Banff' 17 ABMR definition, although this would not alter our conclusions. Finally, we did not assess sera for presence of non-HLA DSA, which could account for some survival heterogeneity within the sABMR'13 group. ${ }^{25}$

In summary, we show that the introduction of C4d-negative ABMR in Banff' 13 increased numbers of ABMR diagnoses and improved the prognostic performance. However, adopting HLA-DSA negative ABMR and eliminating the suspicious category caused reclassification of biopsies towards groups with 
worse or better allograft outcome. Our data demonstrate the clinical relevance of an intermediate category, of which the reintroduction could be considered. This study provides a framework for rapid retrospective evaluation of future changes to the Banff classification.

This article is protected by copyright. All rights reserved 


\section{Contributors}

JC, HA and MN designed the study and the analysis plan. JC, HA, EL, MC, AS, EVL, BS, VVS, MR, VD, OT, DK, MPE and MN were involved in clinical data collection and data quality control. EL and MR performed all biopsy readings from Leuven and Lyon respectively. AS produced and analysed the HLA genotyping and antibody data. JC did the statistical analyses and created the figures and tables, with input from HA and MN. JC, HA and MN wrote the article, and all coauthors revised and approved it.

\section{Acknowledgments}

This work is supported by The Research Foundation Flanders (FWO) and the Flanders Innovation \& Entrepreneurship agency (VLAIO), with a TBM project (grant no. IWT.150199). MN and BS are senior clinical investigators of The Research Foundation Flanders (FWO) (1844019N and 1842919N, respectively). EVL and JC hold a fellowship grant (1143919N and 1196119N, respectively) from The Research Foundation Flanders (FWO). MN is also funded by a C3 internal grant from the KU Leuven (grant no. C32/17/049). OT is supported by the Agence Nationale pour la Recherche (ANR-16-CE17-000701), the Fondation pour la Recherche Médicale (PME20180639518), and the Etablissement Français du Sang.

\section{Disclosure}

The authors of this manuscript have no conflicts of interest to disclose as described by the American Journal of Transplantation.

\section{Data Availability Statement}

The data that support the findings of this study are available from the corresponding author upon reasonable request. 


\section{ORCIDs}

Jasper Callemeyn, http://orcid.org/0000-0003-2235-9842;

Heleen Ameye, https://orcid.org/0000-0002-1057-0352;

Evelyne Lerut, https://orcid.org/0000-0003-3937-7190;

Aleksandar Senev, https://orcid.org/0000-0002-6196-4669;

Maarten Coemans, https://orcid.org/0000-0001-8442-3673;

Elisabet Van Loon, https://orcid.org/0000-0001-9796-9157;

Ben Sprangers, https://orcid.org/0000-0003-1314-9675;

Vicky Van Sandt, https://orcid.org/0000-0002-9748-4069;

Maud Rabeyrin, https://orcid.org/0000-0003-0713-3271;

Valerie Dubois, https://orcid.org/0000-0002-1169-7697;

Olivier Thaunat, https://orcid.org/0000-0002-3648-8963;

Dirk Kuypers, https://orcid.org/0000-0001-5546-9680;

Marie-Paule Emonds, https://orcid.org/0000-0002-2653-8656;

Maarten Naesens, https://orcid.org/0000-0002-5625-0792;

This article is protected by copyright. All rights reserved 


\section{Figure Legend}

Figure 1 Changes in diagnostic category of kidney allograft biopsies according to the Banff'01, Banff' 13 and Banff' 17 classification for antibody-mediated rejection ( $\mathrm{N}=491$ biopsies).

Figure 2 Prognostic performance of the current and previous Banff classifications for ABMR ( $N=893$ transplantations).

Figure 3 Death-censored kidney transplant survival according to changing diagnostic categories between Banff'01 and Banff' 13 (N=893 transplantations).

Figure 4 Death-censored kidney transplant survival according to changing diagnostic categories between Banff' 13 and Banff' 17 (N=893 transplantations).

Figure 5 Prognostic performance of potential alternatives to the Banff' 17 classification. 


\section{Tables}

Table 1 Main demographic and clinical characteristics of the study population ( $\mathrm{N}=949$ transplantations).

Table 2 Changes in diagnostic category of kidney allograft biopsies according to the Banff'01, Banff' 13 and Banff' 17 classification for ABMR.

Table 3 Multivariable hazard ratio for death-censored allograft failure, according to Banff recategorization, transplant glomerulopathy, IFTA and cellular rejection ( $\mathrm{N}=2721$ biopsies).

This article is protected by copyright. All rights reserved 


\section{References}

1. Sellarés J, De Freitas DG, Mengel M, et al. Understanding the causes of kidney transplant failure: The dominant role of antibody-mediated rejection and nonadherence. Am J Transplant. 2012;12(2):388-399. doi:10.1111/j.1600-6143.2011.03840.x

2. El-Zoghby ZM, Stegall MD, Lager DJ, et al. Identifying specific causes of kidney allograft loss. Am J Transplant. 2009;9(3):527-535. doi:10.1111/j.1600-6143.2008.02519.x

3. Van Loon E, Bernards J, Van Craenenbroeck AH, Naesens M. The Causes of Kidney Allograft Failure. Transplantation. 2020;104(2):e46-e56. doi:10.1097/TP.0000000000003012

4. Racusen LC, Colvin RB, Solez K, et al. Antibody-mediated rejection criteria - An addition to the Banff '97 classification of renal allograft rejection. Am J Transplant. 2003;3(6):708-714. doi:10.1034/j.1600-6143.2003.00072.x

5. Haas M. An updated Banff schema for diagnosis of antibody-mediated rejection in renal allografts. Curr Opin Organ Transplant. 2014;19(3):315-322. doi:10.1097/MOT.0000000000000072

6. Haas M. The Revised (2013) Banff Classification for Antibody-Mediated Rejection of Renal Allografts: Update, Difficulties, and Future Considerations. Am J Transplant. 2016;16(5):13521357. doi:10.1111/ajt.13661

7. Haas M. Evolving criteria for the diagnosis of antibody-mediated rejection in renal allografts. Curr Opin Nephrol Hypertens. 2018;27(3):137-143. doi:10.1097/MNH.0000000000000398

8. Roufosse C, Simmonds N, Clahsen-Van Groningen M, et al. A 2018 Reference Guide to the Banff Classification of Renal Allograft Pathology. Transplantation. 2018;102(11):1795-1814. doi:10.1097/TP.0000000000002366

9. Haas M, Sis B, Racusen LC, et al. Banff 2013 meeting report: Inclusion of C4d-negative antibodymediated rejection and antibody-associated arterial lesions. Am J Transplant 2014;14:272-283. doi:10.1111/ajt.12590

10. Lefaucheur C, Loupy A, Vernerey D, et al. Antibody-mediated vascular rejection of kidney allografts: A population-based study. Lancet. 2013;381(9863):313-319. doi:10.1016/S01406736(12)61265-3

11. De Serres SA, Noël R, Côté I, et al. 2013 Banff Criteria for Chronic Active Antibody-Mediated Rejection: Assessment in a Real-Life Setting. Am J Transplant. 2016;16(5):1516-1525. doi:10.1111/ajt.13624

12. Kozakowski N, Herkner H, Böhmig GA, et al. The diffuse extent of peritubular capillaritis in renal allograft rejection is an independent risk factor for graft loss. Kidney Int. 2015;88(2):332-340. doi:10.1038/ki.2015.64

13. Loupy A, Hill GS, Suberbielle C, et al. Significance of C4d Banff scores in early protocol biopsies of kidney transplant recipients with preformed donor-specific antibodies (DSA). Am J Transplant.

This article is protected by copyright. All rights reserved 
2011;11(1):56-65. doi:10.1111/j.1600-6143.2010.03364.x

14. Gupta A, Broin PÓ, Bao Y, et al. Clinical and molecular significance of microvascular inflammation in transplant kidney biopsies. Kidney Int. 2016;89(1):217-225.

doi:10.1038/ki.2015.276

15. Sis B, Jhangri GS, Bunnag S, Allanach K, Kaplan B, Halloran PF. Endothelial gene expression in kidney transplants with alloantibody indicates antibody-mediated damage despite lack of $\mathrm{C} 4 \mathrm{~d}$ staining. Am J Transplant. 2009;9(10):2312-2323. doi:10.1111/j.1600-6143.2009.02761.x

16. Haas M, Loupy A, Lefaucheur C, et al. The Banff 2017 Kidney Meeting Report: Revised diagnostic criteria for chronic active T cell-mediated rejection, antibody-mediated rejection, and prospects for integrative endpoints for next-generation clinical trials. Am J Transplant. 2018;18:293-307. doi:10.1111/ajt.14625

17. Loupy A, Haas M, Solez K, et al. The Banff 2015 Kidney Meeting Report: Current Challenges in Rejection Classification and Prospects for Adopting Molecular Pathology. Am J Transplant. 2017;17(1):28-41. doi:10.1111/ajt.14107

18. Gaston RS, Cecka JM, Kasiske BL, et al. Evidence for antibody-mediated injury as a major determinant of late kidney allograft failure. Transplantation. 2010;90(1):68-74. doi:10.1097/TP.0b013e3181e065de

19. Loupy A, Haas M, Roufosse C, et al. The Banff 2019 Kidney Meeting Report (I): Updates on and clarification of criteria for T cell- and antibody-mediated rejection. Am J Transplant. May 2020:ajt.15898. doi:10.1111/ajt.15898

20. Mengel M, Chan S, Climenhaga J, et al. Banff initiative for quality assurance in transplantation (BIFQUIT): reproducibility of C4d immunohistochemistry in kidney allografts. Am J Transplant. 2013;13(5):1235-1245. doi:10.1111/ajt.12193

21. Senev A, Coemans M, Lerut E, et al. Histological picture of antibody-mediated rejection without donor-specific anti-HLA antibodies: Clinical presentation and implications for outcome. Am J Transplant. 2019;19(3):763-780. doi:10.1111/ajt.15074

22. Senev A, Emonds M, Van Sandt V, et al. Clinical importance of extended second field high-resolution HLA genotyping for kidney transplantation. Am J Transplant. May 2020:ajt.15938. doi:10.1111/ajt.15938

23. Senev A, Lerut E, Van Sandt V, et al. Specificity, strength, and evolution of pretransplant donorspecific HLA antibodies determine outcome after kidney transplantation. Am J Transplant. 2019. doi:10.1111/ajt.15414

24. Schinstock CA, Sapir-Pichhadze R, Naesens M, et al. Banff survey on antibody-mediated rejection clinical practices in kidney transplantation: Diagnostic misinterpretation has potential therapeutic implications. Am J Transplant. 2019. doi:10.1111/ajt.14979

This article is protected by copyright. All rights reserved 
25 Lefaucheur C, Viglietti D, Bouatou Y, et al. Non-HLA agonistic anti-angiotensin II type 1 receptor antibodies induce a distinctive phenotype of antibody-mediated rejection in kidney transplant recipients. Kidney Int. 2019;96:189-201. doi:10.1016/j.kint.2019.01.030

\section{Supporting Information}

Additional supporting information may be found online in the Supporting Information section at the end of the article.

This article is protected by copyright. All rights reserved 
Figure 1 Changes in diagnostic category of kidney allograft biopsies according to the Banff'01, Banff' 13 and Banff' 17 classification for ABMR ( $N=3662$ biopsies).

All post-transplant biopsies were classified according to Banff'01, Banff' 13 and Banff'17. Of these, 3171 biopsies were not considered as SABMR or ABMR in any classification. The evolution of diagnostic categorization of the other 491 biopsies across different Banff versions is depicted. Line colours represent the diagnostic class in the preceding Banff version. ABMR: antibody-mediated rejection, sABMR: suspicious for ABMR. $1^{\text {st: }}$ biopsies fulfilling the first Banff criterion. DSA: anti-HLA donor-specific antibodies, MVI: microvascular inflammation.

\section{Banff'01}

Banff'13

Banff'17

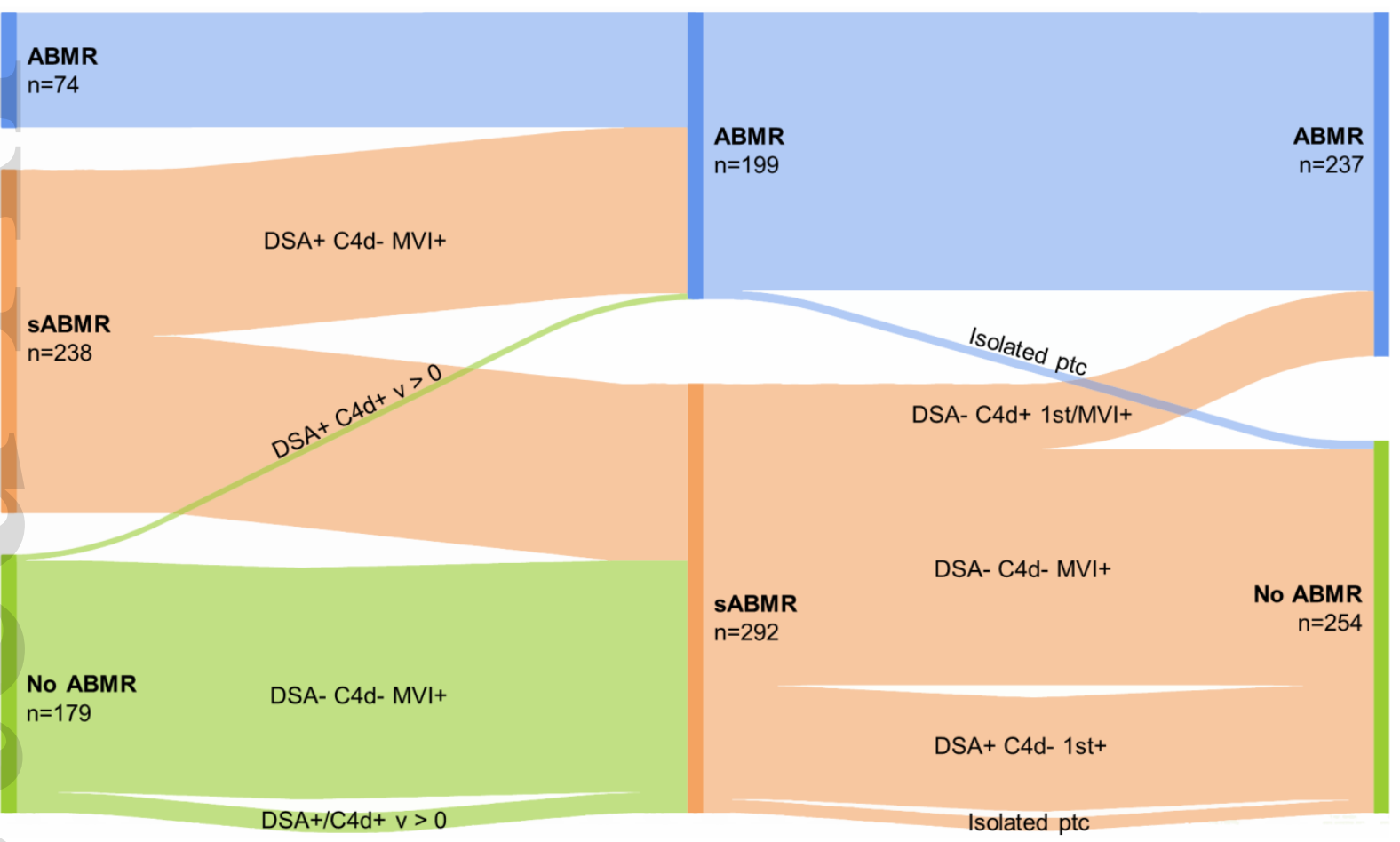

Not considered as SABMR or ABMR in any Banff update $\mathrm{n}=3171$

This article is protected by copyright. All rights reserved 
Figure 2 Prognostic performance of the current and previous Banff classifications for ABMR

A. Patients with a functioning graft at 1 year after transplantation were categorized based on the most severe rejection category (ABMR $>$ sABMR $>$ No ABMR) that occurred in the preceding year. Hazard ratios for death-censored allograft failure were calculated using a Cox proportional hazards model.

B. Harrell's C-statistic for the prediction of death-censored allograft for each Banff iteration at different time points and clinical context. ABMR: antibody-mediated rejection, sABMR: suspicious for ABMR, CI: confidence interval.

A

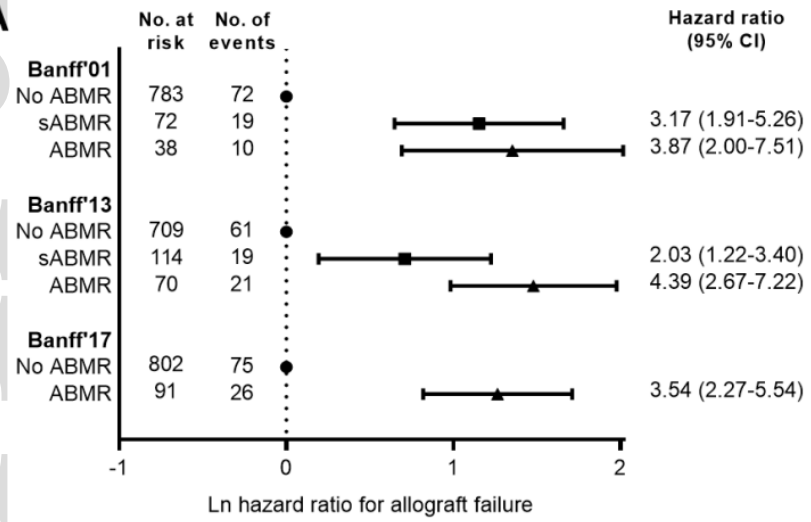

B

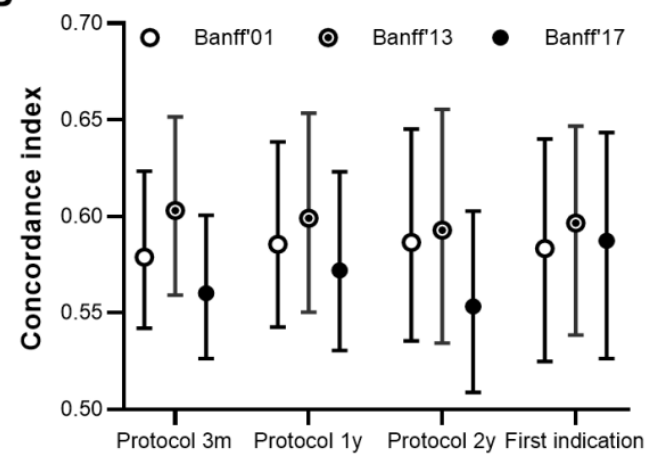


Figure 3 Death-censored kidney transplant survival according to diagnostic reclassification between Banff'01 and Banff'13 (N=893 transplantations).

Kaplan-Meier survival curves of death-censored allograft survival after the first year post transplantation categorized according to the most severe rejection phenotype occurring within the preceding year (ABMR $>$ sABMR > No ABMR). Reported P-values reflect differences between all groups. ABMR: antibodymediated rejection, sABMR: suspicious for ABMR.
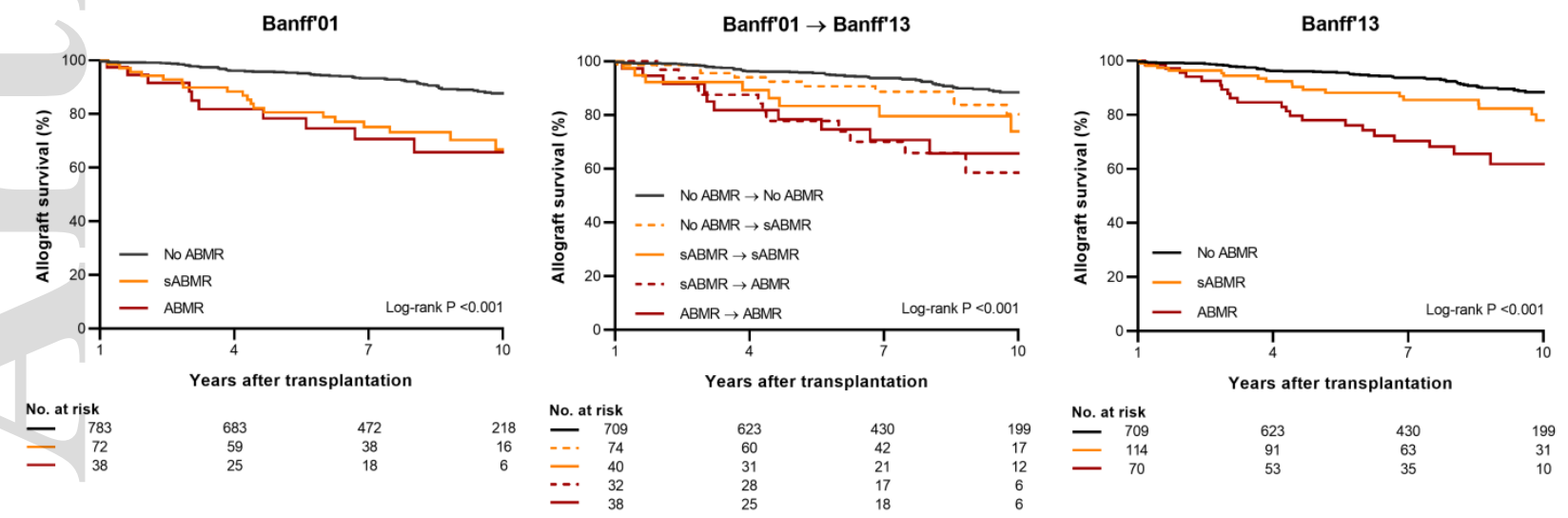
Figure 4 Death-censored kidney transplant survival according to diagnostic reclassification between Banff' 13 and Banff' 17 ( $N=893$ transplantations).

Kaplan-Meier survival curves of death-censored allograft survival after the first year post transplantation categorized according to the most severe rejection phenotype occurring within the preceding year (ABMR $>$ sABMR > No ABMR). Reported P-values reflect differences between all groups. ABMR: antibodymediated rejection, sABMR: suspicious for ABMR.
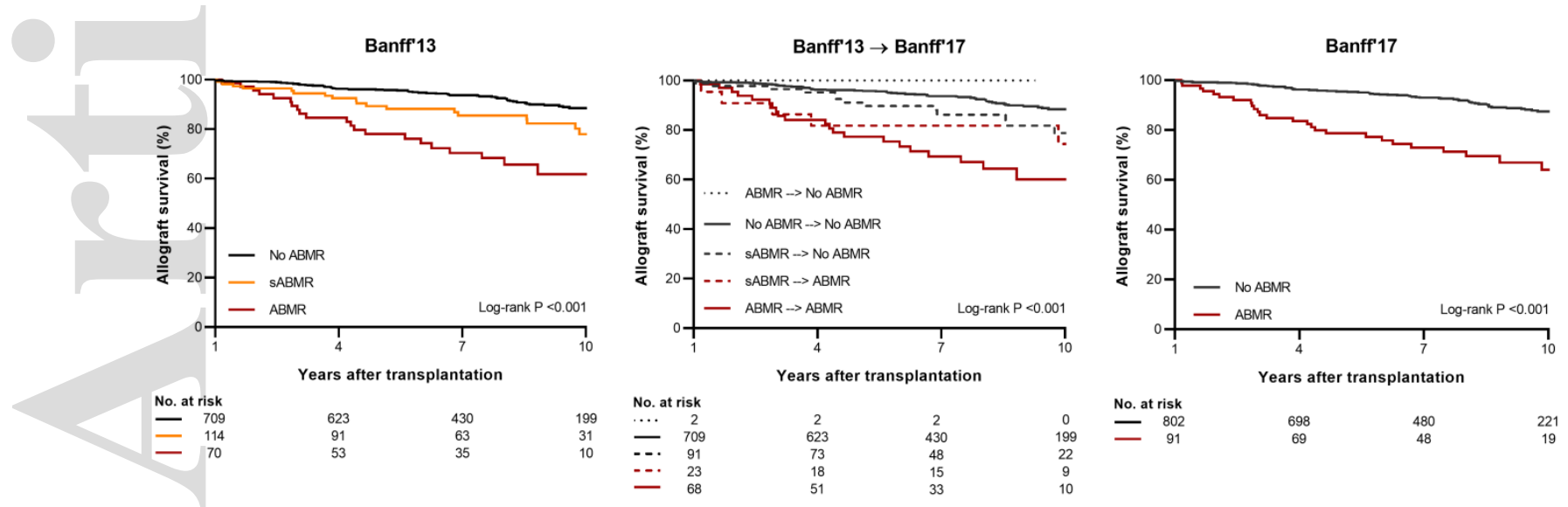


\section{Figure 5 Prognostic performance of potential alternatives to the Banff'17 classification}

A. Landmark survival analysis for death-censored allograft failure, with categorization of transplantations based on the most severe rejection category that occurred in the preceding year. For Alternative 1, the following order was used: $\mathrm{ABMR}^{*}>\mathrm{DSA}$ negative $\mathrm{MVI}>$ No ABMR, for Alternative 2: ABMR* $>$ DSA positive suspicious $>$ DSA negative MVI $>$ No ABMR. ABMR* in the two alternative models indicates DSA positive biopsies that were considered as ABMR in Banff'17. Hazard ratios for death-censored allograft failure were calculated using a Cox proportional hazards model.

B. Harrell's C-statistic for the prediction of death-censored allograft failure at different time points and clinical context. ABMR: antibody-mediated rejection, MVI: microvascular inflammation, DSA: donorspecific antibodies, CI: confidence interval.

C. Kaplan-Meier survival curves of death-censored allograft survival after the first year post transplantation categorized according to the most severe rejection phenotype by Alternative 1 occurring within the preceding year. Reported P-values reflect differences between all groups.

D. Kaplan-Meier survival curves of death-censored allograft survival after the first year post transplantation categorized according to the most severe rejection phenotype by Alternative 2 occurring within the preceding year. Reported P-values reflect differences between all groups.

This article is protected by copyright. All rights reserved 
A

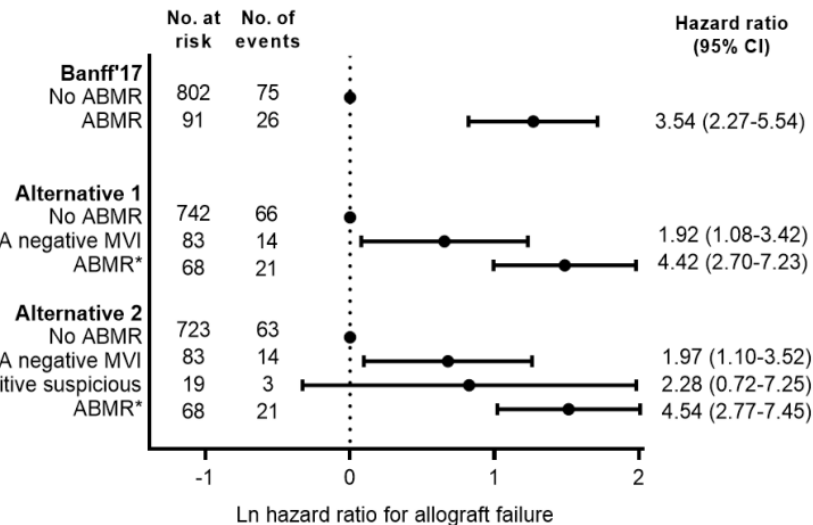

C

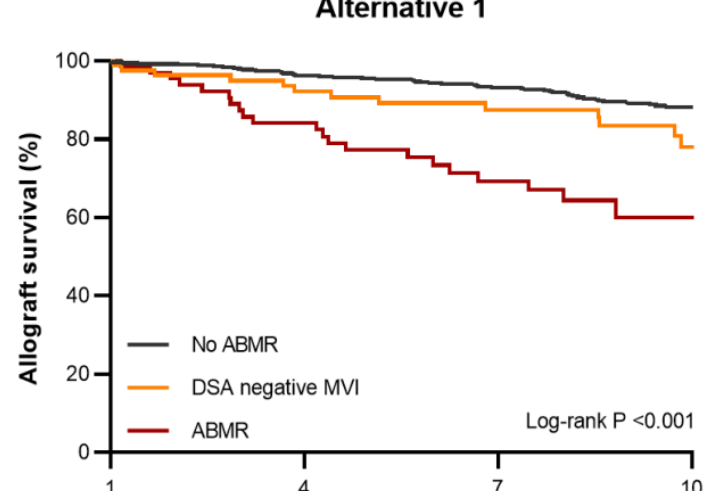

No. at risk Years after transplantation

742
$-\quad 83$
$-\quad 68$
650
66
51
445
50
33

D

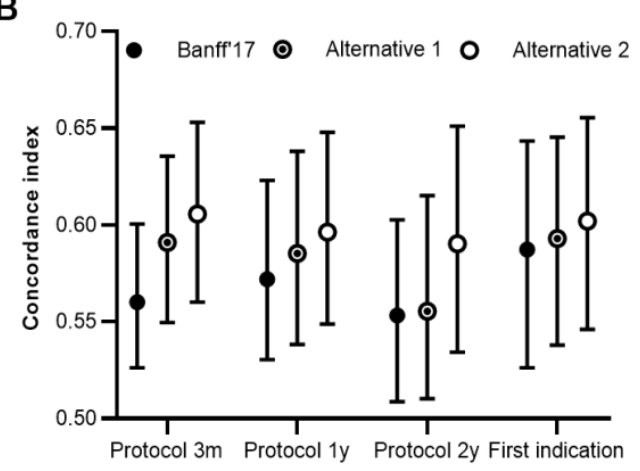

Alternative 2

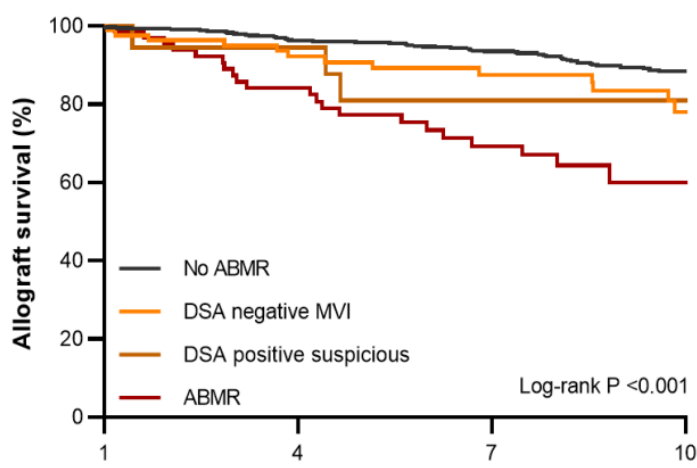

$\begin{array}{cc}\text { No. at risk } \\ - & 723 \\ - & 83 \\ - & 19 \\ - & 68\end{array}$

635438

66

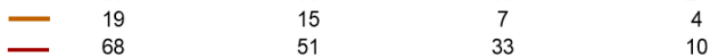

438
50
7
33 
Table 1 Main demographic and clinical characteristics of the study population ( $N=949$ transplantations).

SD: standard deviation, DSA: donor-specific antibodies, TAC: tacrolimus, MPA: mycophenolic acid, CS: corticosteroids

\begin{tabular}{|c|c|}
\hline \multicolumn{2}{|l|}{ Recipients } \\
\hline Age (y), mean \pm SD & $53.6 \pm 13.3$ \\
\hline Sex (male), $\mathrm{n}(\%)$ & $580(61.1)$ \\
\hline Repeat transplantation, $\mathrm{n}(\%)$ & $143(15.1)$ \\
\hline Northern European ethnicity, n(\%) & $933(98.3)$ \\
\hline \multicolumn{2}{|l|}{ Donors } \\
\hline $\operatorname{Age}(y)$, mean \pm SD & $47.7 \pm 14.9$ \\
\hline Sex (male), n(\%) & $505(53.2)$ \\
\hline Deceased donor, $\mathrm{n}(\%)$ & $892(94.0)$ \\
\hline Donation after brain death, $\mathrm{n}(\%)$ & $739(77.9)$ \\
\hline Cold ischemia time $(\mathrm{h})$, mean $+-\mathrm{SD}$ & $14.2 \pm 5.7$ \\
\hline \multicolumn{2}{|l|}{ Immunological profile at the index biopsy } \\
\hline HLA-A/B/DR mismatches, mean \pm SD & $2.71 \pm 1.3$ \\
\hline HLA-A/B/DR/DQ mismatches, mean $\pm \mathrm{SD}$ & $3.19 \pm 1.8$ \\
\hline HLA-A mismatches, mean \pm SD & $0.97 \pm 0.7$ \\
\hline HLA-B mismatches, mean \pm SD & $1.02 \pm 0.6$ \\
\hline HLA- DRB1 mismatches, mean \pm SD & $0.72 \pm 0.6$ \\
\hline HLA- DRB345 mismatches, mean \pm SD & $0.30 \pm 0.5$ \\
\hline HLA-DQA1 mismatches, mean \pm SD & $0.42 \pm 0.5$ \\
\hline HLA- DQB1 mismatches, mean \pm SD & $0.65 \pm 0.6$ \\
\hline HLA- DPA1 mismatches, mean \pm SD & $0.23 \pm 0.4$ \\
\hline HLA- DPB1 mismatches, mean \pm SD & $0.88 \pm 0.7$ \\
\hline Donor-specific HLA antibodies, n(\%) & $145(15.3)$ \\
\hline - $\quad$ Pre-tx DSA Class I, n(\%) & $-\quad 62(6.5)$ \\
\hline - $\quad$ Pre-tx DSA Class II, n(\%) & $-66(7.0)$ \\
\hline - $\quad$ De Novo DSA Class I, n(\%) & $-48(5.1)$ \\
\hline - De Novo DSA Class II, n(\%) & $-\quad 36(3.8)$ \\
\hline $\begin{array}{l}\text { Treatment at the time of transplantation } \\
\text { follow-up }\end{array}$ & \\
\hline
\end{tabular}

This article is protected by copyright. All rights reserved 


\begin{tabular}{|l|c|}
\hline Delayed graft function, $\mathrm{n}(\%)$ & $173(18.2)$ \\
\hline $\begin{array}{l}\text { Immunosuppressive regimen: TAC-MPA-CS, } \\
\mathrm{n}(\%)\end{array}$ & $812(85.6)$ \\
\hline Induction therapy, $\mathrm{n}(\%)$ & $394(41.5)$ \\
\hline
\end{tabular}

This article is protected by copyright. All rights reserved 
Table 2 Changes in diagnostic category of kidney allograft biopsies according to the Banff Banff'01, Banff' 13 and Banff' 17 classification for ABMR.

Only biopsies that were considered as SABMR or ABMR by at least one Banff version are presented. ABMR: antibody-mediated rejection, sABMR: suspicious for ABMR. MVI: microvascular inflammation

\begin{tabular}{|c|c|c|}
\hline Banff'01 Classification & Number & Reason of change classification \\
\hline No ABMR Banff'01 & 179 & \\
\hline$\Rightarrow$ ABMR Banff'13 & 4 & $\mathrm{v}>0$ \\
\hline$\rightarrow$ sABMR Banff'13 & $\begin{array}{l}10 \\
165\end{array}$ & $\begin{array}{l}\mathrm{v}>0 \text { MVI- C4d- DSA+ } \\
4 \mathrm{v}>0 \text { MVI- C4d+ DSA- } \\
161 \text { MVI+ DSA- }\end{array}$ \\
\hline sABMR Banff'01 & 238 & \\
\hline$\rightarrow$ ABMR Banff'13 & 121 & $\mathrm{MVI}+\mathrm{C} 4 \mathrm{~d}-\mathrm{DSA}+$ \\
\hline$\rightarrow$ sABMR Banff'13 & 117 & \\
\hline ABMR Banff'01 & 74 & \\
\hline$\rightarrow$ ABMR Banff'13 & 74 & \\
\hline \multicolumn{3}{|l|}{ Banff'13 Classification } \\
\hline sABMR Banff'13 & 292 & \\
\hline$\rightarrow$ ABMR Banff'17 & 44 & C4d+ DSA- biopsies fulfilling first criterion \\
\hline$\rightarrow$ No ABMR Banff'17 & $\begin{array}{l}79 \\
169\end{array}$ & $\begin{array}{l}\text { C4d- DSA+ biopsies fulfilling first criterion } \\
161 \mathrm{MVI}+\mathrm{C} 4 \mathrm{~d}-\mathrm{DSA}- \\
8 \text { isolated ptc with TCMR/borderline } \\
\text { changes }\end{array}$ \\
\hline ABMR Banff'13 & 199 & \\
\hline$\rightarrow$ ABMR Banff'17 & 193 & \\
\hline$\rightarrow$ No ABMR Banff'17 & 6 & Isolated ptc with TCMR/borderline changes \\
\hline \multicolumn{3}{|l|}{ Banff'17 Classification } \\
\hline ABMR Banff'17 & 237 & \\
\hline No ABMR Banff'17 & 254 & \\
\hline
\end{tabular}


Table 3 Multivariable hazard ratio for death-censored allograft failure, according to Banff recategorization, transplant glomerulopathy, IFTA and cellular rejection ( $\mathrm{N}=2721$ biopsies)

\begin{tabular}{|c|c|c|c|c|}
\hline \multicolumn{5}{|l|}{ Protocol biopsy 3 months } \\
\hline Variable & No. at risk & No. events & HR (95\% CI) & $P$ \\
\hline \multicolumn{5}{|l|}{ Banff'13 $\rightarrow$ '17 change } \\
\hline No $A B M R \rightarrow$ No ABMR & 753 & 71 & 1 & \\
\hline sABMR $\rightarrow$ No ABMR & 41 & 10 & $3.65(1.87-7.11)$ & $<0.001$ \\
\hline $\mathrm{sABMR} \rightarrow \mathrm{ABMR}$ & 6 & 0 & & \\
\hline $\mathrm{ABMR} \rightarrow \mathrm{ABMR}$ & 32 & 14 & $6.88(3.83-12.38)$ & $<0.001$ \\
\hline Cg score & 832 & 95 & $1.14(0.46-2.82)$ & 0.79 \\
\hline IFTA grade & 832 & 95 & $1.58(1.11-2.24)$ & 0.01 \\
\hline \multicolumn{5}{|l|}{ Cellular rejection } \\
\hline No cellular rejection & 703 & 73 & 1 & \\
\hline Borderline changes & 76 & 15 & $1.89(1.08-3.30)$ & 0.03 \\
\hline TCMR & 53 & 7 & $1.19(0.55-2.61)$ & 0.66 \\
\hline \multicolumn{5}{|l|}{ Protocol biopsy 1 year } \\
\hline Variable & No. at risk & No. events & HR (95\% CI) & $P$ \\
\hline \multicolumn{5}{|l|}{ Banff'13 $\rightarrow$ '17 change } \\
\hline No ABMR $\rightarrow$ No ABMR & 695 & 59 & 1 & \\
\hline sABMR $\rightarrow$ No ABMR & 34 & 7 & $2.31(1.03-5.19)$ & 0.04 \\
\hline $\mathrm{sABMR} \rightarrow \mathrm{ABMR}$ & 2 & 0 & & \\
\hline $\mathrm{ABMR} \rightarrow \mathrm{ABMR}$ & 38 & 13 & $4.57(2.44-8.54)$ & $<0.001$ \\
\hline $\mathrm{ABMR} \rightarrow$ No ABMR & 1 & 1 & $8.67(1.05-71.64)$ & 0.05 \\
\hline Cg score & 770 & 80 & $1.73(1.09-2.75)$ & 0.02 \\
\hline IFTA grade & 770 & 80 & $1.52(1.15-2.00)$ & $<0.001$ \\
\hline \multicolumn{5}{|l|}{ Cellular rejection } \\
\hline No cellular rejection & 660 & 62 & 1 & \\
\hline Borderline changes & 75 & 9 & $1.29(0.63-2.64)$ & 0.48 \\
\hline TCMR & 35 & 9 & $1.74(0.80-3.81)$ & 0.16 \\
\hline \multicolumn{5}{|l|}{ Protocol biopsy 2 years } \\
\hline Variable & No. at risk & No. events & HR (95\% CI) & $P$ \\
\hline \multicolumn{5}{|l|}{ Banff'13 $\rightarrow$ '17 change } \\
\hline No ABMR $\rightarrow$ No ABMR & 577 & 44 & 1 & \\
\hline sABMR $\rightarrow$ No ABMR & 40 & 9 & $2.11(1.01-4.42)$ & 0.05 \\
\hline
\end{tabular}

This article is protected by copyright. All rights reserved 


\begin{tabular}{|c|c|c|c|c|}
\hline $\begin{array}{l}\mathrm{sABMR} \rightarrow \mathrm{ABMR} \\
\mathrm{ABMR} \rightarrow \mathrm{ABMR}\end{array}$ & $\begin{array}{l}6 \\
22\end{array}$ & $\begin{array}{l}1 \\
6\end{array}$ & $\begin{array}{l}1.96(0.27-14.35) \\
3.23(1.32-7.94)\end{array}$ & $\begin{array}{l}0.51 \\
\mathbf{0 . 0 1}\end{array}$ \\
\hline Cg score & 645 & 60 & $2.34(1.61-3.39)$ & $<0.001$ \\
\hline IFTA grade & 645 & 60 & $2.05(0.28-14.97)$ & 0.48 \\
\hline $\begin{array}{l}\text { Cellular rejection } \\
\text { No cellular rejection } \\
\text { Borderline changes } \\
\text { TCMR }\end{array}$ & $\begin{array}{l}559 \\
63 \\
23\end{array}$ & $\begin{array}{l}47 \\
8 \\
5\end{array}$ & $\begin{array}{l}1 \\
1.76(0.81-3.84) \\
1.65(0.19-14.39)\end{array}$ & $\begin{array}{l}0.15 \\
0.65\end{array}$ \\
\hline First indication biopsy & & & & \\
\hline Variable & No. at risk & No. events & HR (95\% CI) & $P$ \\
\hline $\begin{array}{c}\text { Banff'13 } \rightarrow \text { '17 change } \\
\text { No ABMR } \rightarrow \text { No ABMR } \\
\text { sABMR } \rightarrow \text { No ABMR } \\
\text { sABMR } \rightarrow \text { ABMR } \\
\text { ABMR } \rightarrow \text { ABMR } \\
\text { ABMR } \rightarrow \text { No ABMR }\end{array}$ & $\begin{array}{l}348 \\
57 \\
20 \\
45 \\
4\end{array}$ & $\begin{array}{l}72 \\
12 \\
6 \\
18 \\
0\end{array}$ & $\begin{array}{l}1 \\
0.91(0.47-1.75) \\
1.28(0.54-3.07) \\
1.91(1.09-3.37)\end{array}$ & $\begin{array}{l}0.77 \\
0.58 \\
\mathbf{0 . 0 3}\end{array}$ \\
\hline Cg score & 474 & 108 & $1.88(1.17-3.03)$ & $<0.001$ \\
\hline IFTA grade & 474 & 108 & $1.71(1.17-2.50)$ & $<0.001$ \\
\hline $\begin{array}{l}\text { Cellular rejection } \\
\text { No cellular rejection } \\
\text { Borderline changes } \\
\text { TCMR }\end{array}$ & $\begin{array}{l}267 \\
49 \\
158\end{array}$ & $\begin{array}{l}53 \\
10 \\
45\end{array}$ & $\begin{array}{l}1 \\
0.77(0.38-1.58) \\
1.26(0.81-1.97)\end{array}$ & $\begin{array}{l}0.48 \\
0.31\end{array}$ \\
\hline $\begin{array}{l}\text { Years after transplantation, } \\
\text { per } 1 \text { year increase }\end{array}$ & 474 & 108 & $1.24(1.04-1.48)$ & 0.02 \\
\hline
\end{tabular}

This article is protected by copyright. All rights reserved 